Association for Information Systems

AIS Electronic Library (AISeL)

Wirtschaftsinformatik Proceedings 2005

Wirtschaftsinformatik

February 2005

\title{
Individualisierte Anlageberatung: Axiomatische Fundierung von Zielfunktionen zur Bewertung von Anlagealternativen
}

Hans Ulrich Buhl

Universität Augsburg

Stefan Volkert

Universität Augsburg

Veronica Winkler

Universität Augsburg

Follow this and additional works at: http://aisel.aisnet.org/wi2005

\section{Recommended Citation}

Buhl, Hans Ulrich; Volkert, Stefan; and Winkler, Veronica, "Individualisierte Anlageberatung: Axiomatische Fundierung von Zielfunktionen zur Bewertung von Anlagealternativen" (2005). Wirtschaftsinformatik Proceedings 2005. 26.

http://aisel.aisnet.org/wi2005/26

This material is brought to you by the Wirtschaftsinformatik at AIS Electronic Library (AISeL). It has been accepted for inclusion in Wirtschaftsinformatik Proceedings 2005 by an authorized administrator of AIS Electronic Library (AISeL). For more information, please contact elibrary@aisnet.org. 
In: Ferstl, Otto K, u.a. (Hg) 2005. Wirtschaftsinformatik 2005: eEconomy, eGovernment, eSociety; 7. Internationale Tagung Wirtschaftsinformatik 2005. Heidelberg: Physica-Verlag

ISBN: 3-7908-1574-8

(C) Physica-Verlag Heidelberg 2005 


\title{
Individualisierte Anlageberatung: Axiomatische Fundierung von Zielfunktionen zur Bewertung von Anlagealternativen
}

\author{
Hans Ulrich Buhl, Stefan Volkert, Veronica Winkler \\ Universität Augsburg
}

Zusammenfassung: Im Finanzdienstleistungsbereich spielt die Individualisierung von Beratungsleistungen eine immer bedeutendere Rolle. Kunden erwarten die individualisierte Leistungserstellung nicht nur im persönlichen Beratungsgespräch, sondern auch über elektronische Kommunikationskanäle. Um die individuelle Beratung auch IT-gestützt vornehmen zu können, ist es vor allem notwendig, neben „,harten“ finanzwirtschaftlichen Zielen wie Rendite und Risiko auch „, weiche" Ziele - wie die Variabilität, Liquidierbarkeit, Verwaltbarkeit und Nachvollziehbarkeit - in die (teil-)automatisierte Beratung mit einzubeziehen. Die bisherigen Ansätze zur systemgestützten Anlageberatung bewerten Anlagealternativen jedoch fast ausschließlich auf Basis etablierter finanzwirtschaftlicher Kennzahlen und lassen somit ,weiche“ Ziele außer Acht. Die Autoren stellen fachlich begründete Axiome auf und leiten daraus Funktionen ab, welche die Bewertung der ,weichen "Ziele einer Anlagealternative erlauben. Die Methodik ist über die Anlageberatung hinaus geeignet, in allgemeinen Beratungssituationen die betrachteten Objekte nicht nur hinsichtlich ,, harter“, sondern auch ,weicher" Ziele beurteilen zu können.

Schlüsselworte: Individualisierung, systemgestützte Anlageberatung, multikriterielles Zielsystem, axiomatische Vorgehensweise, qualitative Ziele

\section{Einleitung}

In den letzten Jahren spielt die Individualisierung von Dienstleistungen im Finanzdienstleistungsmarkt eine immer größere Rolle: Durch das Angebot individueller Lösungen entzieht sich der Finanzdienstleister (FDL) der erhöhten Konkurrenz, da seine Produkte schwerer vergleichbar und damit weniger austauschbar sind. Obwohl diese Anbieterstrategie nicht unbedingt im Interesse des Kunden ist, da auf diesem Wege höhere Preise durchsetzbar sind als auf transparenten Märkten, ist der Kunde i. d. R. trotzdem wesentlich zufriedener, da ihm eine auf ihn zugeschnittene Lösung einen höheren Nutzen stiftet als Standardlösungen und er 
sich durch die individuelle Beratung besser betreut fühlt [FrSt02]. Um Beratungsleistungen in der Anlageberatung individualisiert zu gestalten, müssen sowohl die Bedürfnisse, Wünsche und Interessen des Kunden als auch seine sozialen und finanziellen Restriktionen hinsichtlich der Anlageziele berücksichtigt werden [Buhl ${ }^{+}$03a; Buhl ${ }^{+}$04]. Betrachtet man den Status quo, werden i. A. die Rendite und zum Teil das Risiko der Anlage in die Beratung miteinbezogen. Neben diesen „harten“ Zielen lassen sich in der Literatur noch weitere „weiche“ finden, die für einen Kunden von großer Relevanz bei der Anlageentscheidung sein können und aus diesem Grund bei einer individualisierten Beratung berücksichtigt werden sollten: Dazu gehören die Maximierung der Liquidierbarkeit, der Variabilität, der Verwaltbarkeit und der Nachvollziehbarkeit [Buhl ${ }^{+} 03 \mathrm{~b}$; Hein02; Oeh195; Ruda88; $\left.\mathrm{Voge}^{+} 00\right]$. Dies wird auch durch repräsentative Studien untermauert [IMSI00].

\section{Problemstellung}

Um eine individualisierte Beratung durchführen zu können und damit ein Beratungsergebnis zu erhalten, das die Bedeutung der einzelnen Ziele für den Kunden gemäß Abbildung 1 durch kundenindividuelle Zielgewichtungen berücksichtigt, müssen auf der einen Seite die Zielgewichtungen auf Basis der Einstellungen ${ }^{1}$ des Kunden sowie auf Basis der Einschätzungen des FDL über den Kunden ${ }^{2}$ ermittelt werden [Buhl ${ }^{+}$03a; Buhl $\left.{ }^{+} 04\right]$. Auf der anderen Seite müssen für die potenziellen Anlagealternativen gemäß Abbildung 1 Zielfunktionswerte ( $\mathrm{Zfw}$ ) vorliegen, die widerspiegeln, wie gut die Anlagealternativen den einzelnen Zielen jeweils entsprechen. Die folgende Abbildung 1 veranschaulicht die einer individualisierten Beratung zu Grunde liegenden Komponenten, die im Teilprojekt SIPKIS ${ }^{3}$ des vom bayerischen Wissenschaftsministerium geförderten Forschungsverbunds FORSIP ${ }^{4}$ erarbeitet und anhand des Anwendungsszenarios „Altersvorsorge“ prototypisch umgesetzt werden. Dabei entsprechen die Produktkategorienkombinationen ${ }^{5}$ den Anlagealternativen.

Bedürfnisse, Wünsche und Interessen

soziale und finanzielle Restriktionen und psychische Belastbarkeit

Situierung, Individualisierung und Personalisierung in kundenzentrischen Informationssystemen

4 Bayerischer Forschungsverbund für Situierung, Individualisierung und Personalisierung in der Mensch-Maschine-Interaktion

5 In dieser Arbeit wird vereinfachend davon ausgegangen, dass der individualisierten Beratung keine große Anzahl an Einzelprodukten, sondern Produktkategorien zu Grunde liegen. Eine Produktkategorie $i \in\{1, \ldots, \mathrm{n}\}$ fasst dabei eine Menge von Anlageprodukten zusammen, die der gleichen Produktgattung angehören. Das Ergebnis der Beratung ist schließlich eine Produktkategorienkombination $j$, die sich aus mehreren Produktkategorien gewichtet mit den Anteilsgewichten $a_{i j}$ je Produktkategorie, 


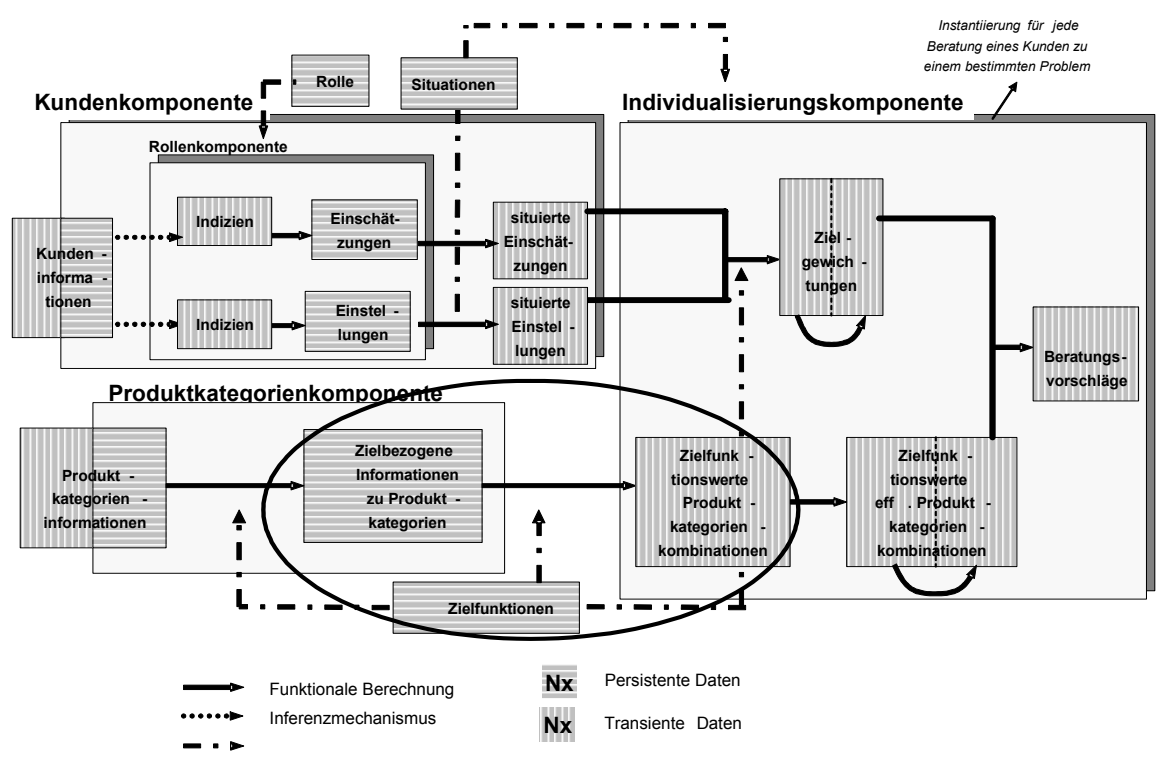

Abbildung 1: Komponenten einer individualisierten Finanzdienstleistungsberatung (Quelle: $\left.\left[\mathrm{Buhl}^{+} 04\right]\right)$

Im Folgenden fokussiert sich die Arbeit im Wesentlichen auf den markierten Bereich des in der Abbildung 1 dargestellten Gesamtkonzepts einer individualisierten Beratung. Auf die in diesem Ausschnitt enthaltenen Elemente wird im weiteren Verlauf genauer eingegangen.

Betrachtungsgegenstand dieser Arbeit sind dabei sowohl Selbstberatungs- als auch beratungsunterstützende Systeme im Anlagekontext. Die grundlegende Frage einer jeden individualisierten Anlageberatung ist, welche PKK aus der Menge effizienter ${ }^{6}$ PKK für den Kunden adäquat sind. Grundlage für die Entscheidung sind, wie oben beschrieben, auf Kundenseite die individuellen Zielgewichtungen und auf Produktseite die Zfw der PKK. Um die Zfw der PKK bestimmen zu können, müssen im ersten Schritt die Ausprägungen - im Folgenden als zielbezogene Informationen (zI) bezeichnet -, die die PK hinsichtlich der Ziele besitzen, ermittelt werden. Die zI zu den in der Beratung betrachteten Zielen - im vorliegenden Fall

und mit $\sum_{i=1}^{n} a_{i j}=1$ zusammensetzt. Produktkategorien werden dabei im Folgenden mit PK, Produktkategorienkombinationen mit PKK abgekürzt.

6 Effizienz wird hier i. S. v. Paretoeffizienz verstanden. Eine PKK ist immer dann in der Menge der effizienten PKK enthalten, wenn keine andere PKK existiert, die bei höchstens gleich hohen Werten der anderen Zfw hinsichtlich eines Zfw einen besseren Wert aufweist. 
einer Anlageberatung Maximierung der Rendite, Minimierung des Risikos, Maximierung der Liquidierbarkeit, der Variabilität, der Nachvollziehbarkeit und der Verwaltbarkeit - lassen sich aus vorliegenden Informationen über die PK wie der Handelbarkeit oder Kurswerten ableiten [Buhl $\left.{ }^{+} 04\right]$. Liegen die zI jeder PK vor, müssen sie entsprechend je Ziel miteinander verrechnet werden, um die Zfw der PKK zu erhalten. Hier ist zu berücksichtigen, dass es sich um eine multikriterielle Entscheidungssituation handelt und die Zfw folglich so bestimmt werden müssen, dass sich die PKK anhand dieser in eine entsprechende Rangfolge bringen lassen. Dabei handelt es sich um eine zweistufige Entscheidung: In einem ersten Schritt sind auf Basis der Zfw die hinsichtlich der relevanten Ziele ineffizienten PKK zu entfernen, da einer unter finanzwirtschaftlichen Gesichtspunkten qualitativ hochwertigen Beratung nur effiziente Anlagealternativen zu Grunde liegen sollen. In einem zweiten Schritt sind die für den Kunden adäquaten PKK zu identifizieren. Welche Arten von Funktionen sich für die Berechnung der Zfw eignen, soll in der vorliegenden Arbeit diskutiert werden. Dies entspricht dem in der Abbildung 1 dargestellten Schritt von den zI der PK zu den Zfw der PKK.

Im Folgenden wird zunächst ein kurzer Überblick über die bestehende Literatur zu multikriteriellen Entscheidungsproblemen und dabei eingesetzten Verfahren gegeben. Im Kapitel 0 der Arbeit wird die Eignung unterschiedlicher Verrechnungsfunktionen für die Berechnung der Zfw im Rahmen einer Anlageberatung diskutiert. Dabei wird wie folgt vorgegangen: Nach der Definition des jeweiligen Ziels werden die für die Berechnung des Zfw notwendigen Axiome aufgestellt und begründet. Die axiomatische Methode hat den Vorteil, dass man bereits vor der Verwendung überlegt, welche Eigenschaften man für die spezielle Verwendung fordert und nicht bereits durch die Wahl eines konkreten Operators andere, unter Umständen wesentlich geeignetere, Eigenschaften außer Betracht lässt. Anschließend werden aus den Axiomen in Frage kommende Verrechnungsfunktionen abgeleitet.

\section{Literaturüberblick}

Bisher werden in der Literatur vor allem folgende Aspekte betrachtet: Für die quantitativen Ziele Rendite und Risiko existieren in der Portfoliotheorie bereits etablierte finanzwirtschaftliche Kennzahlen und Verfahren zur Verrechnung einzelner Rendite- und Risikowerte zu einem aggregierten Wert [BrMy03; Gerk01; Mark59; StBr02]. Für die qualitativen Ziele müssen demgegenüber noch geeignete Verrechnungsfunktionen ausgewählt werden. Dabei ist die vorliegende Problemstellung sehr ähnlich zu der Auswahl der Verrechnungsfunktionen für die Aktualisierung von Einstellungen und Einschätzungen: Sowohl bei den Einstellungen respektive Einschätzungen und den Kundeninformationen, aus denen sie abgeleitet werden, als auch den zI über die PK und damit den Zfw der PKK handelt es sich 
um unsicheres Wissen: In Abhängigkeit davon, wie hoch die Qualität der Datenquelle (z. B. Datenbanken, Beratungsgespräch, Webtracking) ist, aus der die Kundeninformationen stammen, wird festgelegt, mit welcher Gewissheit die daraus gewonnene Einstellung des Kunden respektive Einschätzung des FDL über den Kunden auch tatsächlich auf den Kunden zutrifft. Auch auf der Produktseite liegt unsicheres Wissen vor, da es sich bei den zI der PK um Durchschnittswerte handelt, die aus Daten unterschiedlicher Quellen (z. B. onvista, Schätzwerte von Analysten) abgeleitet werden. Aus diesem Grund sollen bei der Berechnung sowohl der Einstellungen/Einschätzungen als auch der Zfw Verfahren aus dem Bereich der Fuzzy-Theorie eingesetzt werden [Maye ${ }^{+}$94; Wern84; Zade65; Zimm92]. Da es sich hierbei um die Verrechnung unsicheren Wissens handelt und die Forschung zu Problemstellungen der Fuzzy-Theorie bereits anerkannte Leistungen erbracht hat, werden die dort erarbeiteten Ergebnisse im Folgenden zu Grunde gelegt, um unter Verwendung einer axiomatischen Vorgehensweise geeignete Funktionen zur Ermittlung der Zfw von PKK zu identifizieren.

\section{Auswahl der Funktionen zur Berechnung der Zielfunktionswerte der Produktkategorienkombinationen}

Im Folgenden werden für die einzelnen Ziele entsprechende Funktionen zur Ermittlung der Zfw der PKK ausgewählt. Für alle Ziele berechnet sich der gesuchte $\mathrm{Zfw}$ der PKK $P K K_{j}$ aus den einzelnen, in der jeweiligen Kombination enthaltenen, zI der PK $i$ hinsichtlich des jeweiligen Ziels. Die zI der PK für die Ziele Rendite und Risiko liegen definitionsgemäß im Intervall $[0 ; \infty) .^{7}$ Dabei gilt für die Rendite: Je höher die zI der PK, umso mehr entspricht die PK der Zielsetzung. Für das Risiko trifft der umgekehrte Fall zu. Für die Ziele Liquidierbarkeits-, Variabilitäts-, Verwaltbarkeits- und Nachvollziehbarkeitsmaximierung sind die Werte der $\mathrm{zI} \in[0,1]$. Dabei werden für Rendite und Risiko lediglich die bereits aus der Portfoliotheorie bekannten finanzwirtschaftlichen Kennzahlen vorgestellt. Für die anderen Ziele werden im ersten Schritt wünschenswerte Axiome festgelegt, denen die Funktionen zur Berechnung der Zfw genügen sollen. Anschließend werden die auf Basis der Axiome in Frage kommenden Funktionen aufgeführt und die für die vorliegende Problemstellung adäquate Alternative ausgewählt.

\footnotetext{
Negative Werte werden bei der Rendite ausgeschlossen, da PK mit einer erwarteten negativen Rendite nicht in die Beratung mit einbezogen werden.
} 


\subsection{Rendite und Risiko}

Die erwartete jährliche Rendite $\mu_{P K K j}$ einer PKK $j$ aus den mit dem jeweiligen Anteil $x_{i j}$ gewichteten Renditen $\mu_{i}$ der PK $i$ wird gemäß der folgenden Formel berechnet [Gerk01, S. 1699]:

$$
\mu_{P K K_{j}}=\sum_{i=1}^{n} x_{i j} \mu_{i}
$$

Risiko wird , [...] als Abweichen von geplanten Größen in beide Richtungen [...]“ [StBr02, S. 54] verstanden. Die Bemessung des Risikos einer PK erfolgt in der überindividuellen Betrachtung über die Varianz $\sigma_{i}^{2}$. Daraus berechnet sich die Varianz einer Lösungsalternative $\sigma_{P K K j}{ }^{2}$ gemäß folgender Gleichung:

$$
\sigma_{P K K_{j}}^{2}=\sum_{i=1}^{n} \sum_{k=1}^{n} x_{i j} x_{k j} C O V_{i k}
$$

Dabei bezeichnet $C O V_{i k}$ die Kovarianz zwischen der PK $i$ und der PK $k \in \mathrm{N}$ [StBr02]. ${ }^{8}$ Dementsprechend sind die Varianzen der einzelnen PK und deren Kovarianzen zu ermitteln.

\subsection{Liquidierbarkeit}

Wie bereits dargestellt, lässt sich neben den traditionell relevanten Anlagezielen „Renditemaximierung“ und „Risikominimierung“ in der Literatur das Ziel „Maximierung der Liquidierbarkeit“ finden. Dort wird darunter die Möglichkeit zur täglichen und möglichst kostengünstigen Liquidierbarkeit des angelegten Vermögens verstanden:

- „Die Liquidität einer Kapitalanlage ist als Möglichkeit für den Anleger zu verstehen, bestehende Anlagen jederzeit zu fairen Preisen verkaufen zu können.“ [StBr02, S. 74]

- „Die Liquidität umfaßt also zwei grundlegende Eigenschaften von Wertpapieren, die miteinander eng zusammenhängen. Es sind dies die jederzeite Handelbarkeit (Zeitdimension) und der geringe Preiseinfluß (Preisdimension). Ein perfekt liquides Wertpapier kann jederzeit ohne Kurseinfluß ge- und verkauft werden. ..." [Kemp98].

$8 \quad$ Bei der Berechnung der Varianz der PKK ist zu beachten, dass die Kovarianz einer PK zu sich selbst $C O V_{i i}$ der Varianz der PK $\sigma_{i}^{2}$ entspricht. 
Nach der Klärung des Begriffs wird nun eine Funktion $f:[0,1]^{n} \rightarrow[0,1]$ gesucht, die den einzelnen zI der PK einen aggregierten $\mathrm{Zfw}$ für das Liquidierbarkeitsziel zuweist und den im Folgenden aufzustellenden Axiomen genügt.

(A1) Die Bedeutung, die die einzelnen zI der PK für den Zfw der PKK haben, ist abhängig vom Anteil der PK an der PKK. Dies entspricht der natürlichen Vorstellung, dass nur der Anteil, den die PK an der PKK besitzt, so liquide ist wie die PK. Eine Anforderung an die Zielfunktion ist hiermit, dass sie eine Gewichtung der Zfw erlaubt. Formal bedeutet dies, dass neben den zI der PK auch deren Anteile an den PKK als Argumente in die Funktion $f$ eingehen.

(A2) Die in den aggregierten Zfw eingehenden zI der PK sind zum Teil sehr widersprüchlich. Das Ergebnis der Verrechnung sollte dabei eine Art „tradeoff" zwischen diesen darstellen. Grund hierfür ist, dass sich die Liquidierbarkeit der PKK zwischen der PK, die mit der geringsten zI eingeht und der PK mit der höchsten zI liegen sollte. Um dieser Anforderung gerecht zu werden, muss die Zielfunktion die Mittelwerteigenschaft erfüllen [Acze61, S. 162; Maye ${ }^{+} 94$, S. 46; Zimm92, S. 42].

D. h. für alle $x, y \in[0 ; 1]$ gilt:

$\min (x, y) \leq \mathrm{f}(x, y) \leq \max (x, y)$.

(A3) Je höher die zI der in der PKK enthaltenen PK sind, desto höher soll auch der aggregierte Zfw der PKK sein. Diese Forderung sorgt für ein Entsprechen der menschlichen Erwartung, dass die Konjunktion von einzelnen PK mit hoher Liquidierbarkeit (in Abhängigkeit von ihrem Anteil), zu einer hohen aggregierten Liquidierbarkeit der PKK führt. Es besteht demgemäß eine Forderung nach monotonem Verhalten.

$f$ ist monoton wachsend in jede Richtung, d. h. für jedes $u, x, v, y \in[0,1]$ gilt:

Wenn $u \leq x$ und $v \leq y$, so $f(u, v) \leq f(x, y)$.

(A4) Die Reihenfolge, in der die zI der PK verrechnet werden, soll keine Rolle spielen. Dies entspricht der intuitiven Erwartung, dass das Ergebnis für den Zfw einer PKK unabhängig von der Reihenfolge, in der die zI eingehen, gleich sein soll. Gefordert wird folglich Kommutativität:

$f$ ist kommutativ, d. h. für jedes $x, y \in[0,1]$ gilt:

$f(x, y)=f(y, x)$.

(A5) Eine weitere Forderung an die Zielfunktion ist ihre Stetigkeit. Diese Forderung sorgt dafür, dass kleine Änderungen der zu aggregierenden zI auch zu kleinen Änderungen im Aggregat führen. Gefordert wird also:

$f$ ist stetig in beiden Variablen. 
(A6) Zusätzlich soll die Funktion folgendes Verhalten aufweisen: Gehen PK mit gleichen zI in die Zielfunktion ein, entspricht das Ergebnis der zI der PK. Dies entspricht dem natürlichen Verhalten, dass die Aggregation von PK mit gleichen zI hinsichtlich der Liquidität - gewichtet mit ihrem Anteil - dazu führt, dass das Ergebnis gleich deren Liquidierbarkeit ist. Gefordert ist also idempotentes Verhalten:

$f$ ist idempotent, d. h. für jedes $z \in[0,1]$ gilt:

$f(z, z)=z$.

Nachdem die Axiome festgelegt wurden, sollen im nächsten Schritt geeignete Funktionen zur Verrechnung der zI ausgewählt werden. Analysiert man mögliche Funktionen hinsichtlich der o. g. Anforderungen, so ergeben sich als Alternativen für die Zielfunktion Operatoren aus der Klasse der Mischnormen (Durchschnittsoperatoren) [Maye ${ }^{+}$94; Wern84; Zimm92]. Mischnormen liegen in ihrem Ergebnis zwischen dem Minimum- und dem Maximum-Operator und damit zwischen der Und- und der Oder-Verknüpfung von einzelnen Werten [Maye ${ }^{+}$94, S. 42]. Während für den Minimum-Operator immer die kleinste eingehende zI der in der PKK enthaltenen PK das Ergebnis bestimmt, tritt beim Maximum-Operator der gegenteilige Fall ein. Die Durchschnittsoperatoren, die zwischen diesen beiden Operatoren anzusiedeln sind, entsprechen damit der oben geforderten Mittelwerteigenschaft. An dieser Stelle ist noch eine weitere Anforderung an den Operator zu stellen: Der Operator sollte parametrisierbar sein. Grund ist, dass eine der größten Schwierigkeiten bei Entscheidungssituationen, in denen die in Betracht kommenden Alternativen hinsichtlich verschiedener Kriterien in eine Rangfolge gebracht werden sollen, die Auswahl des geeigneten Operators ist, d. h. ob der Operator eher dem logischen Und oder dem logischen Oder nahe kommen soll. Je nach Entscheidungssituation kann ein anderer Operator geeignet sein. Dies ist für den praktischen Einsatz jedoch kaum handhabbar [Wern87, S. 297]. Aus diesem Grund existieren parametrisierbare Operatoren (der Parameter wird im Folgenden mit $\gamma$ bezeichnet), deren Nähe zum Und bzw. Oder sich durch die Wahl des Parameterwerts entsprechend variieren lässt. Des Weiteren geht aus empirischen Untersuchungen hervor, dass parametrisierbare Operatoren in ihren Ergebnissen menschliche Beurteilungen von Alternativen wesentlich besser nachempfinden als nicht parametrisierte [ZiZy80; ZiZy83].

In Tabelle 1 werden gebräuchliche Durchschnittsoperatoren aufgeführt, die obigen Anforderungen genügen. Einzige Ausnahme stellt der $\gamma$-Operator dar, der keine Idempotenz und in Teilbereichen keine Mittelwerteigenschaft besitzt [Wern84, S. 161 f.]. Da dieser Operator aber in der anschließenden Diskussion im Vergleich der Eignung der Operatoren für den praktischen Einsatz und damit der Auswahl der Verrechnungsfunktion für das Liquiditätsziel eine wichtige Rolle spielt, wird er mit aufgelistet. 


\begin{tabular}{|l|l|l|}
\hline Operator & Definition $(\gamma \in[0,1])$ & \\
\hline Fuzzy-And & $\gamma \min (x, y)+\frac{(1-\gamma)(x+y)}{2}$ & $(3)$ \\
\hline Fuzzy-Or & $\gamma \max (x, y)+\frac{(1-\gamma)(x+y)}{2}$ & $(4)$ \\
\hline min-max-Operator & $\gamma \min (x, y)+(1-\gamma) \max (x, y)$ & $(5)$ \\
\hline Algebraic-Prod-Sum & $\gamma x y+(1-\gamma)(x+y-x y)$ & $(6)$ \\
\hline \hline$\gamma$-Operator & $(x y)^{1-\gamma}(1-(1-x)(1-y))^{\gamma}$ & $(7)$ \\
\hline
\end{tabular}

Tabelle 1: Eine Auswahl gebräuchlicher zweistelliger Durchschnittsoperatoren (vgl. [Maye+94, S. 44-45; Wern84, S. 161-164; Zimm92, S. 36-38])

Um aus den nach Anwendung der Axiome verbleibenden Durchschnittsoperatoren einen auszuwählen, kann nicht mehr ausschließlich formal vorgegangen werden, da alle diese Funktionen, bis auf den $\gamma$-Operator, den o. g. Axiomen entsprechen. Neben den mathematischen Anforderungen lassen sich jedoch noch weitere Aspekte nennen, die für die Auswahl des Operators eine wichtige Rolle spielen [Maye ${ }^{+}$94, S. 48-49; Zimm92, S. 39-43]. Für den hier vorliegenden Verwendungszweck - Bewertung einer PKK in einer Beratung bei einem FDL hinsichtlich des Ziels „Maximierung der Liquidierbarkeit“ - sind insbesondere die folgenden Aspekte von großer Bedeutung:

- Empirische Relevanz

- Mathematische Attraktivität

Unter der empirischen Relevanz ist zu verstehen, wie gut der Operator geeignet ist, menschliches Verhalten abzubilden. Dabei basieren die folgenden Aussagen bezüglich der Eigenschaften der Operatoren hinsichtlich dieses Kriteriums auf den Ergebnissen empirischer Tests, die in den jeweils genannten Literaturquellen dargestellt sind. In den Tests wird im ersten Schritt anhand eines t-Tests überprüft, ob die Abweichungen der Ergebnisse der Operatoren von den empirischen Ergebnissen rein zufälligen Schwankungen unterliegen. Trifft dies zu, liegt für die Operatoren eine der Realität angemessene Aggregation vor. Damit kann aber noch keine Aussage über die Güte der ermittelten Werte der Operatoren getroffen werden. Hierzu wird der mittlere prognostische Fehler, der die Abweichung der Ergebnisse der Operatoren von den empirischen Werten misst, für jeden der Operatoren berechnet. Je kleiner der prognostische Fehler ist, desto besser entspricht der Operator dem tatsächlichen menschlichen Verhalten [Wern84, S. 175-179]. Gemäß den in [Wern84, S. 176-178, 186] dargestellten Testergebnissen, die entsprechend der oben beschriebenen Vorgehensweise ermittelt wurden, eignet sich der $\gamma$-Operator 
für die Abbildung menschlichen Entscheidungsverhaltens im Allgemeinen besser als der min-max-Operator und der Algebraic-Prod-Sum Operator. So ist ein großer Nachteil des min-max-Operators, dass nicht effiziente Lösungen die gleiche Bewertung erhalten können wie effiziente [Wern87, S. 197]. Grund hierfür ist u. a., dass nur die extremen Werte (Minimal- und Maximalwerte) der zI der PK berücksichtigt werden. Für den $\gamma$-Operator lässt sich sagen, dass seine guten Testergebnisse insbesondere darauf basieren, dass der Operator durch geeignete Wahl des Parameters $\gamma$ den Rahmenbedingungen der jeweiligen Entscheidungssituation flexibel und damit bestmöglich angepasst werden kann. Laut Werners wird der $\gamma$-Operator jedoch bei einer Entscheidungssituation - wie sie hier vorliegt -, in der mehr als zwei unterschiedlich gewichtete Kriterien aggregiert werden sollen, hinsichtlich der empirischen Relevanz noch durch die parametrisierten Operatoren Fuzzy-Und und Fuzzy-Oder übertroffen [Wern84, S. $179+$ S. 191]. Setzt man diese Operatoren gemeinsam bei einer solchen Problemstellung ein, ergibt sich eine noch höhere Übereinstimmung des Ergebnisses, d. h. der auf Basis des Operators ermittelten Werte, mit dem Ergebnis der Realität, d. h. der Bestimmung der Werte durch Personenbefragung. Dieses positive Resultat beruht darauf, dass man je nach Art der zu aggregierenden Kriterien zwischen den zwei sich ergänzenden Operatoren Fuzzy-Und und -Oder wählen kann und nicht auf einen Operator festgelegt ist. Ein weiterer Vorteil dieser Operatoren ist, dass die Qualität der Ergebnisse dieser beiden Operatoren nicht sehr sensibel auf eine Fehlbestimmung von $\gamma$ reagiert [Wern84, S. 191].

Die mathematische Attraktivität bezieht sich demgegenüber auf den zu leistenden Rechenaufwand und damit bspw. die Performanz eines Systems. Hierbei ergibt sich laut Werners ein Vorteil der Operatoren min-max, Fuzzy-Und und FuzzyOder gegenüber dem $\gamma$-Operator aufgrund dessen nicht-linearer Struktur [Wern87, S. 297].

Aus dieser Diskussion wird deutlich, dass sich sowohl hinsichtlich der empirischen Relevanz als auch der mathematischen Attraktivität die Operatoren FuzzyUnd und Fuzzy-Oder am besten für den praktischen Einsatz eignen. Die sich daraus ergebenden Fragen sind jedoch, welcher der beiden Operatoren einzusetzen ist und wie der geeignete $\gamma$-Wert gefunden werden kann. Zielsetzung im vorliegenden Entscheidungsproblem ist die Ermittlung der Liquidierbarkeit einer PKK aus den zI der in ihr enthaltenen PK gewichtet mit ihrem Anteil. Hierbei handelt es sich eher um eine Und-Verknüpfung als um eine Oder-Verknüpfung, da nicht eine der zI das Ergebnis bestimmen soll, sondern alle. Folglich ist das Fuzzy-Und einzusetzen. Welchen Wert $\gamma$ annehmen soll, lässt sich pauschal nicht beantworten. Dieser sollte in Abhängigkeit von der tatsächlichen Entscheidungssituation, in der der Operator eingesetzt wird, bestimmt werden. Dabei lassen sich folgende Richtlinien für die Wahl eines Wertes von $\gamma$ nennen: Für $\gamma=1$ entspricht das Ergebnis des Fuzzy-Und dem min-Operator, für $\gamma=0$ dem arithmetischen Mittel. D. h. für den ersten Fall bestimmt die schlechteste zI das Ergebnis. Je mehr man sich 0 an- 
nähert, desto stärker wird die kompensatorische Wirkung. Die gesuchte Funktion für die Liquidierbarkeit hat folgendes Aussehen:

$$
f\left(x_{1}, \ldots, x_{n}, a_{1}, \ldots, a_{n}\right)=\gamma \min \left(x_{1}, \ldots, x_{n}\right)+(1-\gamma) \sum_{i=1}^{n} a_{i} x_{i}
$$

Die in der Tabelle 1 enthaltene Ausgangsformel für das Fuzzy-Und wurde durch die Formel (8) in einer allgemeinen Form dargestellt, die die Berücksichtigung von n statt zwei zI $x_{i}$ der PK und Gewichtungsfaktoren $a_{i}$ als Anteil der PK an der PKK ermöglicht.

\subsection{Variabilität}

Neben der Möglichkeit, das angelegte Vermögen früher als geplant in Anspruch zu nehmen, ist ein weiterer Aspekt für die Flexibilität der Anlage u. a. auch die Einflussnahme auf weitere Parameter der Anlage - im Folgenden als Variabilität bezeichnet -, wie die Möglichkeit zur Variation der Höhe und Häufigkeit der Sparbeiträge [IMSI00]. Dabei können Liquidierbarkeit und Variabilität für den Kunden von sehr unterschiedlicher Relevanz sein: So kann für einen Kunden, der in Kürze eine Immobilie erwerben möchte, die Liquidierbarkeit des Vermögens sehr wichtig sein. Wenn aber nicht mit einer positiven oder negativen Veränderung der Einkommenssituation zu rechnen ist, da der Kunde bspw. Beamter ist, ist für ihn dagegen die Möglichkeit zur Variation der Höhe und Häufigkeit der Sparbeiträge von geringer Bedeutung.

Dabei entspricht der Wertebereich der Variabilität dem Intervall [0,1]: 1 steht für perfekt variable PK, 0 für das Gegenteil. Gesucht wird auch hier eine Funktion $g:[0,1]^{n} \rightarrow[0,1]$, die den einzelnen zI aus den Anlageformen ein aggregiertes Variabilitätsziel zuweist.

Die Wirkung der Kombination von PK bezüglich der Variabilität kann analog zur Liquidierbarkeit angenommen werden. Auch bei der Variabilität ist der Anteil, den die PK an der PKK besitzt, zu berücksichtigen. Der sich aus den zI ergebende $\mathrm{Zfw}$ soll des Weiteren ebenfalls zwischen der minimal und der maximal eingehenden zI der PK liegen. Gefordert werden also von $g$ die gleichen Eigenschaften wie von der Verrechnungsfunktion $f$ für die Liquidierbarkeit: Mittelwerteigenschaft, Kommutativität, Monotonie, Stetigkeit und Idempotenz. Zusätzlich gelten auch hier die gleichen Aussagen bezüglich der empirischen Relevanz und der mathematischen Attraktivität, wodurch sich als Verrechnungsfunktion ebenfalls die erweiterte Fuzzy-Und Funktion (8) ergibt. 


\subsection{Verwaltbarkeit}

Mit der Verwaltbarkeit soll der Verwaltungsaufwand erfasst werden, den eine PKK beim Kunden generiert. Der Verwaltungsaufwand setzt sich aus zwei Bestandteilen zusammen: Zum Ersten entsteht einmaliger Verwaltungsaufwand bei der erstmaligen Anlage in den vorgeschlagenen PK. Hierzu gehören z. B. die Formalitäten bei der Konto- bzw. Depoteröffnung wie die Legitimationsprüfung und das Ausfüllen des Fragebogens nach dem Wertpapierhandelsgesetz. Zum Zweiten entsteht laufender Verwaltungsaufwand durch die Notwendigkeit, dass ein Kunde seine PKK während der Laufzeit beobachtet und ggf. Transaktionen vornimmt. Dazu gehören z. B. Entgegennahme von Kontrollmitteilungen, Umschichtungen, Wiederanlagen nach Laufzeitende, Anlage von Ausschüttungen usw. Anlageformen, die ein Ruhenlassen und Abwarten bis zum Planungshorizont erlauben, sind bezüglich der Verwaltbarkeit gegenüber Anlageformen mit häufiger Kundeninteraktion im Vorteil. Der mit einer Kapitalanlage verbundene Verwaltungsaufwand lässt sich Stand heute nicht durch etablierte Verfahren quantifizieren [Buhl $\left.{ }^{+} 03 b\right]$. Ersatzweise soll die Verwaltbarkeit für eine einzelne PK von Experten im normierten Intervall $[0,1]$ bewertet werden. Dabei steht eine Verwaltbarkeit in Höhe von 0 für maximalen Verwaltungsaufwand, eine Verwaltbarkeit von 1 steht für minimalen Verwaltungsaufwand. Zwischenwerte repräsentieren entsprechend abgestuften Verwaltungsaufwand. Die Verwaltbarkeit der PKK aggregiert sich aus den zI über die Verwaltbarkeit der einzelnen Produkategorien. Analog wird auch die Verwaltbarkeit der PKK im Intervall $[0,1]$ mit der o. a. Semantik codiert.

Gesucht wird also eine Funktion $h:[0,1]^{n} \rightarrow[0,1]$, die den zI der PK einen aggregierten Zfw zuweist.

Folgende Eigenschaften soll die gesuchte Aggregationsfunktion $h$ erfüllen:

(B1) Der Verwaltungsaufwand, den eine PK zum Verwaltungsaufwand der PKK beisteuert, soll unabhängig von der Größe des relativen Anteils sein. Für diese Forderung spricht, dass die Verwaltungstätigkeiten für eine Anlageform bereits ab dem ersten Euro anfallen und dann als fix über den Anlagebetrag gesehen werden können. Allerdings findet dann die ebenfalls plausible Annahme, dass man sich umso ausführlicher bzw. sorgfältiger mit den Verwaltungsaufgaben einer Anlageform befasst, je höher deren Anteil am Gesamtdepot ist, keine Berücksichtigung. Jedoch ist dies kein gravierender Nachteil, da das Depot über die relativen Anteile der einzelnen Anlageformen optimiert wird und somit auch kleine relative Anteile, je nach Größe des Gesamtdepots, für große absolute Anlagevolumina stehen können. Diese erfordern dann selbstverständlich ebenfalls hohen Zeitaufwand. Damit ist die Unabhängigkeitsannahme von der relativen Depotgröße gerechtfertigt. Die Verwaltbarkeit aggregiert sich also aus der (Einzel-)Verwaltbarkeit aller Anlageformen, die einen positiven relativen Anteil an der vorgeschlagenen 
PKK aufweisen. Formal bedeutet dies, dass im Gegensatz zu den in den Abschnitten 0 und 4.3 behandelten Zielfunktionen die Anteile $a_{i}$ nicht als Argumente der gesuchten Funktion $h$ vorkommen.

(B2) Je größer die zI bezüglich der Verwaltbarkeit der einzelnen PK, desto größer soll auch die Verwaltbarkeit der PKK sein. Diese natürliche Forderung sorgt dafür, dass große Inputfaktoren zu großem Output führen. Gefordert wird also Monotonie:

$h$ ist monoton wachsend in jede Richtung, d. h. für jedes $u, x, v, y \in[0,1]$ gilt:

Wenn $u \leq x$ und $v \leq y$, so $h(u, v) \leq h(x, y)$.

(B3) Die zI der PK sollen vertauschbar sein. Gefordert wird also Kommutativität:

$h$ ist kommutativ, d. h. für jedes $x, y \in[0,1]$ gilt: $h(x, y)=h(y, x)$.

(B4) zI der PK sollen zu Zwischenaggregaten zusammenfassbar sein. Gefordert wird daher Assoziativität:

$h$ ist assoziativ, d. h. für jedes $x, y, z \in[0,1]$ gilt:

$h(x, h(y, z))=h(h(x, y), z)$.

Aus Kommutativität und Assoziativität lässt sich die Reihenfolgeunabhängigkeit bei der Verrechnung folgern, d. h. es gilt $h(h(x, y), \mathrm{z}))=h(h(x, \mathrm{z}), y)$.

Mit der Eigenschaft der Reihenfolgeunabhängigkeit sind erhebliche rechentechnische Vorteile verbunden. Die eigentlich benötigte n-stellige $(n>2)$ Funktion für $n$ PK kann durch eine $n$-fache Hintereinanderschaltung einer zweistelligen Funktionen ersetzt werden.

(B5) Wenn ein Aggregationselement eine Verwaltbarkeit von 0 aufweist, dies ist gleichbedeutend mit idealtypisch angenommenem ,maximalen Verwaltungsaufwand“, soll unabhängig von der Höhe der weiteren zu verrechnenden Verwaltungsaufwände auch die aggregierte Verwaltbarkeit den Minimalwert annehmen. Das Axiom regelt das Verhalten des Operators am unteren Rand des Intervalls. Hier kommt in einer speziellen Formulierung die zu Grunde liegende intuitive Vorstellung einer UND-Verknüpfung der zI zum Ausdruck. Es soll immer der ungünstigere Verwaltbarkeitswert das Ergebnis bestimmen. Gefordert wird also, dass 0 neutrales Element ist, d. h. für jedes $x \in[0,1]$ gilt $h(x, 0)=h(0, x)=0$.

(B6) Wenn bei der Aggregation eine PK mit der Verwaltbarkeit 1, gleichbedeutend mit ,die Anlageform bringt keinen Verwaltungsaufwand mit sich“ auftritt, soll sich die Verwaltbarkeit des Aggregats aus dem anderen Element bestimmen. Die Bedingung regelt das Verhalten am oberen Rand des Inter- 
valls. Auch hier ist die intuitive Erwartung an die UND-Verknüpfung der zI der PK formuliert, dass Anlageformen, die keinen Verwaltungsaufwand erzeugen, die aggregierte Verwaltbarkeit nicht beeinflussen. Gefordert wird also, dass 1 Identitätselement ist, d. h. für jedes $x \in[0,1]$ gilt $h(x, 1)=h(1, x)=x$.

Funktionen mit diesen Eigenschaften werden als Trianguläre Normen oder kurz T-Normen [ScSk83, S. 73] bezeichnet. T-Normen sind die Funktionenklasse für Konjunktionen in der mehrwertigen Logik. Einzelne Eigenschaften und Kombinationen der Bedingungen für T-Normen wurden vielfältig untersucht [Acze61; ScSk83; DuPr80; Mari00]. Analog zur Diskussion in Abschnitt 0 sollen auch hier nur parametrisierte Funktionen verwendet werden, da sie eine Anpassung an die Problemdomäne erlauben. In der Literatur werden insbesondere nachfolgende parametrisierte T-Normen (s. Tabelle 2) diskutiert:

\begin{tabular}{|l|l|l|l|}
\hline Name & Definition & $\begin{array}{l}\text { Wertebereich } \\
\text { Parameter }\end{array}$ & \\
\hline Yager & $1-\min \left(1, \sqrt[p]{(1-x)^{p}+(1-y)^{p}}\right)$ & $p>0$ & $(9)$ \\
\hline Schweizer & $\sqrt[p]{\max \left(0, x^{p}+y^{p}-1\right)}$ & $p>0$ & $(10)$ \\
\hline Hamacher & $\frac{x y}{p+(1-p)(x+y-x y)}$ & $p \geq 0$ & $(11)$ \\
\hline Frank & $\log _{p}\left(1+\frac{\left(p^{x}-1\right)\left(p^{y}-1\right)}{p-1}\right)$ & $p>0, p \neq 1$ & $(12)$ \\
\hline Dombi & $1-\frac{p}{\left(\frac{1-x}{x}\right)^{p}+\left(\frac{1-y}{y}\right)^{p}}$ & $p>0$ & $(13)$ \\
\hline
\end{tabular}

Tabelle 2: Gebräuchliche parametrisierte T-Normen (vgl. [Maye 94, S. 39; Zimm92, S. 34])

Die bislang diskutierten axiomatischen Anforderungen an T-Normen sind wenig spezifisch, sodass, wie aus den Beispielen in den Tabellen zu sehen, eine große Menge an Funktionen in Frage kommt. Um die Menge einzugrenzen und das Verhalten genauer zu spezifizieren, soll die gesuchte Funktion noch weitere Eigenschaften aufweisen:

(B7) Die Aggregationsfunktion soll stetig sein. Diese Forderung sorgt dafür, dass kleine Änderungen der zu aggregierenden Werte auch zu kleinen Änderun- 
gen im Aggregat führen. Die Stetigkeitsforderung entspricht der intuitiven Erwartung an die Aggregation, da „sprunghaftes“" Verhalten ausgeschlossen wird. Gefordert wird also Stetigkeit von $h$ in allen Variablen.

(B8) Abgesehen von den Grenzwerten, die bereits gesondert behandelt wurden, soll bei der Verknüpfung der zI der sich ergebende Zfw für Verwaltbarkeit immer unterhalb der einzelnen zI liegen. Hier kommt die Überlegung zum Ausdruck, dass der zusätzliche Verwaltungsaufwand jeder weiteren zu berücksichtigenden PK die Verwaltbarkeit der PKK vermindert. Gefordert wird also zusammen mit den bisherigen Eigenschaften archimedisches Verhalten:

$h$ ist eine archimedische T-Norm, d. h. h ist stetig und für jedes $x \in(0,1)$ gilt:

wenn $0<x<1$, so $h(x, x)<x$.

Die oben gefasste Forderung nach archimedischen Verhalten soll noch verallgemeinert werden, sodass man zur Klasse der strikten T-Normen gelangt:

(B9) Statt der bisherigen Monotonie soll nun zusätzlich zu den anderen Eigenschaften von T-Normen die Eigenschaft der strengen Monotonie gefordert werden. Diese Eigenschaft sorgt für Eineindeutigkeit zwischen dem Aggregationsergebnis und den Aggregationselementen. Dies ist nützlich für die Erklärung von aggregierten Ergebnissen, da der Input eindeutig zurückverfolgt werden kann.

Auf Grund der strengen Monotonieeigenschaft gilt dann für jedes $u, v, w, x \in(0,1]:$

wenn $h(u, v)=h(u, w)$ dann $v=w$ und wenn $h(u, v)=h(w, v)$ dann $u=w$.

und

wenn $u<x$ und $v<y$ dann $h(u, v)<h(x, y)$.

T-Normen, die die Eigenschaften Stetigkeit und strenge Monotonie aufweisen, werden auch als strikte T-Normen bezeichnet.

Nach [ScSk83, S. 66] ist jede strikte T-Norm auch archimedisch, sodass das Archimedie-Axiom bei der weiteren Betrachtung außer Acht gelassen werden kann.

Die aus den Axiomen eindeutig ableitbare rationale Funktion ist die parametrisierte Hamacher T-Norm (siehe Gl. (11)).

Nach Mizumoto [Mizu89, S. 223] ist der Hamacher-Operator die einzige strikte TNorm, die als rationale Funktion geschrieben werden kann. Die Darstellbarkeit von $h$ als rationale Funktion ist für die Implementierung wünschenswert. Als rationale Funktion kann $h$ durch Angabe endlich vieler Koeffizienten bzw. endlich vieler Funktionswerte eindeutig bestimmt werden. Aufgrund dieser besonderen 
Stellung innerhalb der Klasse der gesuchten strikten T-Normen soll die HamacherT-Norm als Aggregationsoperator für die Verwaltbarkeit verwendet werden.

\section{Parametrisierung der Hamacher-T-Norm}

Über den Parameter $p$ lässt sich einstellen, wie stark sich die Abweichung der Verwaltbarkeit einzelner PK vom Ideal 1 auf die Verwaltbarkeit der PKK auswirkt. Es lässt sich leicht zeigen, dass höhere Werte für $p$ niedrigere Funktionswerte bei $h(x, y)$ für $x, y \in(0,1)$ erzeugen. Die Verwaltbarkeit einer PKK wird also umso besser bewertet, je niedriger $p$ gewählt wird. Für $p=1$ nimmt der Operator die Form des algebraischen Produkts an. Durch die Wahl des Parameters lässt sich das Aggregationsverhalten also noch für die Fachdomäne adjustieren.

\subsection{Nachvollziehbarkeit}

Kunden haben normalerweise ein besonderes Interesse, das Zustandekommen der Entscheidung für eine PKK zu verstehen. Es entsteht dabei Aufwand für den Kunden, sich mit den in den Kombinationen enthaltenen Anlageformen auseinander zu setzen und deren Funktionsweise gedanklich zu durchdringen. Dieser Aufwand kann zum einen einmalig während der Einarbeitung in eine PK anfallen. Zum anderen aber auch kontinuierlich, für den Fall, dass sich Rahmenbedingungen ändern. Hierzu zählt insbesondere die steuerliche Behandlung von PK. Als Beispiel kann die im Alterseinkünftegesetz für 2005 beschlossene Besteuerung von Lebensversicherungen genannt werden. Kunden werden deshalb in der Regel Anlageformen, die als unkompliziert und leicht zu verstehen gelten, bei denen man sich also bei Entscheidungen nicht gezwungenermaßen auf Fachleute verlassen muss, anderen, komplizierteren Anlageformen vorziehen.

Das zugehörige Anlageziel soll im Folgenden als Nachvollziehbarkeit bezeichnet werden. Ebenso wie bei den Zielen Maximierung von Liquidierbarkeit, Variabilität und Verwaltbarkeit existieren auch für die Nachvollziehbarkeit keine etablierten Kennzahlen, die PK mit ihren zI bewerten. In der Praxis wird auch hier eine Expertenbeurteilung vorgenommen [Ruda88, S. 182; Voge ${ }^{+} 00$, S. 116 ff.]. Ebenso wie bei der Verwaltbarkeit wird auch die Nachvollziehbarkeit in einem Intervall $[0,1]$ bewertet. 1 bezeichnet dabei PK, die perfekt nachvollziehbar sind, den anderen Grenzfall bildet die 0, die für extrem schlechte Nachvollziehbarkeit steht.

Gesucht wird also eine Funktion $k:[0,1]^{n} \rightarrow[0,1]$, die den einzelnen zI aus den Anlageformen einen aggregierten Zfw der PKK zuweist.

Die Wirkung der Kombination von PK bezüglich der Nachvollziehbarkeit kann analog zur Verwaltbarkeit angenommen werden. Berücksichtigt werden nur Anlageformen, die einen positiven relativen Anteil am Gesamtdepot aufweisen. Jede in 
die PKK aufgenommene Anlageform, die in der Nachvollziehbarkeit einen Wert für die zI von $<1$ aufweist, soll den aggregierten $\mathrm{Zfw}$ vermindern. Gefordert werden also von $k$ die mit den Eigenschaften der Aggregationsfunktion für die Verwaltbarkeit identischen Eigenschaften Assoziativität, Kommutativität, Stetigkeit, strenge Monotonie, 0 als neutrales Element, 1 als Identitätselement. Wenn dann noch zusätzlich, wie in 0, aus pragmatischen Erwägungen die Eigenschaft der Darstellbarkeit als rationale Funktion gefordert wird, ergibt sich auch für $k$ eindeutig die Hamacher-T-Norm (s. Gl. (11)) als Aggregationsfunktion.

\section{Zusammenfassung und Ausblick}

Ziel des Aufsatzes war es, ein Konzept vorzustellen, das es erlaubt, in einem Mehrkanalumfeld IT-gestützt Anlagevorschläge aus PKK nicht nur hinsichtlich der zwei üblichen quantitativen Ziele Rendite und Risiko, sondern zusätzlich bezüglich der für viele Kunden ebenfalls wichtigen qualitativen Ziele Liquidierbarkeit, Variabilität, Verwaltbarkeit und Nachvollziehbarkeit zu bewerten. Die Kernaufgabe bestand darin, für jedes Ziel eine geeignete Aggregationsfunktion zu bestimmen, die aus den zI der einzelnen PK den Gesamtwert für die jeweilige Zielfunktion festlegt. Um Beliebigkeit bei der Auswahl der verfügbaren Funktionen zu vermeiden wurde axiomatisch vorgegangen. Aus den fachlich begründeten Axiomen konnten die Aggregationsfunktionen bzw. -funktionsklassen abgeleitet werden. Dabei konnte für die Ziele Verwaltbarkeit und Nachvollziehbarkeit eindeutig der aus der mehrwertigen Logik bekannte Hamacher-Operator abgeleitet werden. Bei den Zielen Liquidierbarkeit und Variabilität wurde die aus den Axiomen ableitbare Klasse der Durchschnittsoperatoren durch zusätzliche qualitative Anforderungen weiter eingeschränkt. Dabei hat sich eine erweiterte Funktion des aus der Fuzzy-Entscheidungstheorie bekannten Fuzzy-Und-Operators anderen Durchschnittsoperatoren als überlegen erwiesen. Die Zielfunktionen werden in der folgenden Tabelle im Überblick dargestellt: 


\begin{tabular}{|l|l|l|}
\hline Name & Definition & $\begin{array}{l}\text { Werte- } \\
\text { bereich } \\
\text { Para- } \\
\text { meter }\end{array}$ \\
\hline Hamacher & $\frac{x y}{p+(1-p)(x+y-x y)}$ & $\mathrm{p} \geq 0$ \\
\hline $\begin{array}{l}\text { Fuzzy- } \\
\text { Und }\end{array}$ & $f\left(x_{1}, \ldots, x_{n}, a_{1}, \ldots, a_{n}\right)=\gamma \min \left(x_{1}, \ldots, x_{n}\right)+(1-\gamma) \sum_{i=1}^{n} a_{i} x_{i}$ & $0 \leq \mathrm{p} \leq 1$ \\
\hline
\end{tabular}

Tabelle 3: Überblick über die Zielfunktionen zur Berechnung der Zfw der PKK

Mit den Ergebnissen der Arbeit sind die Voraussetzungen für den nächsten Schritt in der individuellen Anlageberatung erfüllt: Die Zfw der PKK werden nun verwendet, um die ineffizienten PKK zu entfernen. Abgeschlossen wird die individualisierte Beratung durch die Auswahl einer PKK, deren Zfw mit den Zielgewichtungen des Kunden abgeglichen werden.

Die Anwendung der Methodik beschränkt sich aber im Teilprojekt SIPKIS des Forschungsverbunds FORSIP nicht nur - wie in Abbildung 1 dargestellt -auf die Bewertung der PKK hinsichtlich der weichen Ziele Variabilität, Liquidierbarkeit, Nachvollziehbarkeit und Verwaltbarkeit. Vielmehr wird die entwickelte Methodik - teilweise sogar mit gleichen Funktionen - auch zur Ermittlung der Einstellungen des Kunden und der Einschätzungen des FDL über den Kunden herangezogen [Volk04]. Dabei werden die erarbeiteten Ergebnisse im Rahmen des genannten Forschungsverbundes durch den Einsatz im dort entwickelten Prototypen empirisch überprüft.

Insgesamt konnte im vorliegenden Aufsatz eine Methodik erarbeitet werden, die über die IT-gestützte, individualisierte Anlageberatung hinaus für eine Vielzahl von Anwendungsbereichen, in denen mehrere „weiche“ und „harte“ Ziele gleichzeitig berücksichtigt werden müssen, einsetzbar ist. Voraussetzungen für den erfolgreichen Einsatz der Methodik sind zum einen die Bereitschaft zur Nutzung der elektronischen Transaktionskanäle durch den Berater oder Kunden und eine Transaktionsfrequenz, die ein „Kennenlernen“ des Kunden und eine systematische Erfassung der Einstellungen bzw. Einschätzungen und die Ableitung der Zielgewichte über einzelne, eher zufällige „Snapshots“ hinaus ermöglicht.

Vor allem bei Beratungen im Multikanalumfeld liegt meist ein mehrdimensionales Zielsystem vor, wobei häufig einige der Ziele von eher „weichem“ Charakter im o. g. Sinn sind. So lässt sich in der Reisebranche eine vergleichbare Problemstellung finden: Das Überleben eines Reisebüros hängt aufgrund der steigenden Transparenz der Angebote durch die Online-Agenturen auch in dieser Branche stark davon ab, den Kunden durch individuelle Angebote besser zu betreuen, als 
dies online möglich ist. Dabei spielen auch hier harte Ziele, wie die Kostenminimierung, insbesondere aber auch weiche Ziele wie die Maximierung des Komforts eine Rolle. IT-Unterstützung ist zum einen schon allein aufgrund der Unüberschaubarkeit des Angebots notwendig. Zusätzlich erwarten viele Kunden heutzutage über verschiedene Kanäle mit ihrem Reisebüro kommunizieren zu können.

Unter den o. g. Einschränkungen für den sinnvollen Einsatz ist die Methodik über den Beratungskontext hinaus allgemein auf Anwendungsbereiche übertragbar, in denen menschliches Entscheidungsverhalten bei der Bewertung komplexer Sachverhalte durch Anwendungssysteme nachgebildet bzw. unterstützt werden soll.

\section{Literatur}

[Acze61] Aczél, J.: Über Funktionalgleichungen und ihre Anwendungen. Birkhäuser Verlag: Basel, 1961.

[BrMy03] Brealey, R. A.; Myers, S. C.: Principles of Corporate Finance. 7. Aufl., McGraw Hill: New York et al., 2003.

[Buhl $\left.{ }^{+} 03 \mathrm{a}\right]$ Buhl, H. U.; Fridgen, M.; Volkert, S.: Systemunterstützt individualisierte Kundenansprache in der Mehrkanalwelt der Finanzdienstleistungsbranche - Repräsentation der Einstellungen von Kunden in einem Kundenmodell. In: Uhr, W.; Esswein, W.; Schoop, E. (Hrsg.) Wirtschaftsinformatik 2003. Band II, Physica-Verlag: Heidelberg, 2003, S. 201-229.

[Buhl ${ }^{+}$03b] Buhl, H. U.; Fridgen, M.; Kaiser, M.: Individualisierte Finanzdienstleistungsberatung: Wirtschaftlichkeit durch Prozessstandardisierung am Beispiel der Altersvorsorge. Erscheint in: Sokolovsky, Z.; Löschenkohl, S. (Hrsg.) Industrialisierung in der Finanzwirtschaft. Gabler: Wiesbaden, 2004.

[Buhl ${ }^{+}$04] Buhl, H. U.; Heinrich, B.; Steck, W.; Winkler, V.: Konzept zur individualisierten Finanzdienstleistungsberatung für Privatkunden und dessen prototypische Umsetzung. Erscheint in: Wirtschaftsinformatik 46/6, 2004.

[DuPr80] Dubois, D.; Prade, H.: Fuzzy Sets and Systems - Theory and Applications. Academic Press: New York et al., 1980.

[FrSt02] Fridgen, M.; Steck, W.: Customer Tracking in the Internet: New Perspectives on Web Site Controlling. Quarterly Journal of Electronic Commerce 3, 2002: S. 235-245.

[Gerk01] Gerke, W.: Portfoliotheorie. In: Gerke, W.; Steiner, M. (Hrsg.) Handwörterbuch des Bank- und Finanzwesens. 3. Aufl., Schäffer-Poeschel: Stuttgart, 2001, S. 16941707.

[Hein02] Hein, J. A.: Präferenzmessung von Anlegern - Verfahren der Entscheidungsunterstützung im Portfoliomanagement und in der Anlageberatung. Lang: Frankfurt am Main et al., 2002. 
[IMSI00] Infratest Burke Finanzforschung; Media Markt Analysen; Sinus Sociovision; ISBA: Soll und Haben 5. Studie durchgeführt im Auftrag von SPIEGEL-Verlag (Hrsg.). SPIEGEL-Verlag: Hamburg, 2000.

[Kemp98] Kempf, A.: Was messen Liquiditätsmaße? DBW 58, 1998: S. 299-311.

[Mark59] Markowitz, H. M.: Portfolio Selection - Efficient Diversification of Investments. John Wiley: New York, 1959.

[Mari00] Marichal J.-L.: On the associativity functional equation. Fuzzy Sets and Systems 114, 2000: S. 381-389.

[Maye ${ }^{+}$94] Mayer, A.; Mechler, B.; Schlindwein, A.; Wolke, R.: Fuzzy Logic - Einführung und Leitfaden zur praktischen Anwendung. Addison-Wesley Publishing Company: Bonn, 1994.

[Mizu89] Mizumoto, M.: Pictorial representations of fuzzy connectives, Part I: cases of Tnorms, T-conorms and averaging operators. Fuzzy Sets and Systems 31, 1989: S. $217-242$.

[Oeh195] Oehler, A.: Die Erklärung des Verhaltens privater Anleger - theoretische Ansätze und empirische Analysen. Schäffer-Poeschel: Stuttgart, 1995.

[Ruda88] Ruda, W.: Ziele privater Kapitalanleger. Gabler: Wiesbaden, 1988.

[ScSk83] Schweizer, B.; Sklar, A.: Probabilistic Metric Spaces. North-Holland: New York et al., 1983.

[StBr02] Steiner, M.; Bruns, C.: Wertpapiermanagement. 8. Aufl., Schäffer-Poeschel: Stuttgart, 2002.

[Volk04] Volkert, S.: Wissensrepräsentation in Customer Relationship ManagementAnwendungssystemen und ökonomische Analysen. Dissertation, Universität Augsburg, 2004

[Voge ${ }^{+}$00] Vogelsang, D.; Sachs, P.; Uppena, J. M.; Oehme, M.; Liebing, R.; Knorr, K.: Handbuch Finanz- und Vermögensgestaltungsberatung - Neues Geschäftsfeld für steuer- und rechtsberatende Berufe. Schäffer-Poeschel: Stuttgart, 2000.

[Wern84] Werners, B.: Interaktive Entscheidungsunterstützung durch ein flexibles mathematisches Programmierungssystem. Minerva Publikation: München, 1984.

[Wern87] Werners, B.: An interactive fuzzy programming system. Fuzzy Sets and Systems 23, 1987: S. 131-147.

[Zade65] Zadeh, L. A.: Fuzzy Sets. Information and Control 8, 1965: S. 338-353.

[Zimm92] Zimmermann, H.-J.: Fuzzy set theory and its applications. 2. Aufl., Kluwer Academic Publishers: Boston et al., 1992.

[ZiZy80] Zimmermann, H.-J.; Zysno, P.: Latent connectives in human decision making. Fuzzy Sets and Systems 4, 1980: S. 37-51.

[ZiZy83] Zimmermann, H.-J.; Zysno, P.: Decisions and evaluations by hierarchical aggregation of information. Fuzzy Sets and Systems 10, 1983: S. 243-260. 